\title{
ON THE CLASSIFICATION OF PERIODIC FLOWS
}

\author{
GIAN-CARLO ROTA ${ }^{1}$
}

1. Introduction. Let $(S, \Sigma, \mu)$ be a probability space, ${ }^{2}$ and let $\phi$ and $\phi^{\prime}$ be measure-preserving transformations of $(S, \Sigma, \mu)$, that is, functions from $\Sigma$ to $\Sigma$ such that $\phi(E) \in \Sigma$ if $E \in \Sigma$ and $\mu(\phi(E))=\mu(E)$. One of the central questions of ergodic theory is: when are $\phi$ and $\phi^{\prime}$ conjugate? We recall that two measure-preserving transformations $\phi$ and $\phi^{\prime}$ are said to be (algebraically) conjugate (cf. Halmos [2, p. 44]) when $\phi^{\prime}=\alpha \circ \phi \circ \alpha^{-1}$ for some transformation $\alpha$ such that both $\alpha$ and its inverse $\alpha^{-1}$ are measure-preserving transformations. We are considering here, for greater generality, set transformations of the Boolean $\sigma$-algebra $\Sigma$, which may or may not be induced by point transformations of the set $S$ (cf. Halmos [2, pp. 42-45]).

To answer this question, several conjugacy invariants of measurepreserving transformations have been introduced, such as the spectrum of the induced unitary operator, entropy, etc. However, these invariants are at present very far from being a complete set, which would allow an immediate decision on whether or not two measurepreserving transformations are conjugate.

Another approach to the question consists in relating a measurepreserving transformation to some one among a set of "canonical forms," selected for their simplicity. The result of Halmos and von Neumann (cf. Halmos [2, p. 48]) goes far in this direction: it is shown that an ergodic measure-preserving transformation with discrete spectrum is conjugate to a rotation on a compact abelian group.

The result of the present note lies in this second direction: we shall prove that every periodic group of measure-preserving transformations, or "flow," ergodic or not, is conjugate to a "restriction" of the simplest such flow, namely, the flow obtained on the lateral surface of unit area of a cylinder by rotating the cylinder around its axis.

2. Definitions. A periodic flow (or simply flow) is a semigroup $\phi_{t}$, $0 \leqq t \leqq \infty$, of transformations $\phi_{t}$ of $\Sigma$ into $\Sigma$, such that $\mu\left(\phi_{t}(E)\right)=\mu(E)$ for every $E$ in $\Sigma$ and for every $t, 0 \leqq t \leqq \infty$. We shall assume the semigroup property $\phi_{t}\left(\phi_{s}(E)\right)=\phi_{t+s}(E)$, and the periodicity condition $\phi_{0}=\phi_{1}$. Clearly, some sort of smoothness condition relative to $t$ of

Received by the editors June $7,1961$.

${ }^{1}$ Work done under contract with the Office of Naval Research.

2 We follow the notation in Dunford-Schwartz [1]. Thus $S$ is a set, $\Sigma$ is a $\sigma$-field (or $\sigma$-algebra) of "measurable" subsets of $\Sigma$, and $\mu$ is a measure defined on sets of $\Sigma$ such that $\mu(S)=1$. 
the flow must be assumed. We shall make the weakest such assumption, as follows. Consider the measure space $\left(C, \Sigma_{1}, \nu\right)$ obtained by taking the product of the given measure space $(S, \Sigma, \mu)$ with the interval $[0,1]$, endowed with ordinary Lebesgue measure. A point of $C$ can be written in the form $(t, s)$, where $0 \leqq t \leqq 1$ and $s \in S$. For $E \in \Sigma$, let $E_{1}$ be the set $\left\{(t, s) \mid s \in \phi_{t}(E), 0 \leqq t \leqq 1\right\}$. Our smoothness assumption is that $E_{1}$ shall be a measurable set, namely, that $E_{1} \in \Sigma_{1}$, for every $E \in \Sigma$. In other words, as $t$ runs from 0 to $1, \phi_{t}(E)$ describes a measurable set in the product.

The above description of a periodic flow can be summarized as follows: a periodic flow is a measurable action of the circle group on a probability space. Any continuous action of the circle group on any topological space which is also endowed with a finite regular measure will satisfy our requirements; it is thus easy to produce "many" examples of periodic flows, of the most disparate varieties.

It follows from the definition that $\phi_{t}^{-1}$ is well-defined, and since $\phi_{t} \circ \phi_{1-t}=I$, that $\phi_{t}^{-1}=\phi_{1-t}$. Defining $\phi_{t}=\phi_{1-t}$ for $t<0$, we can obtain a group defined for $-\infty<t<\infty$.

Two flows $\phi_{t}$ and $\phi_{t}^{\prime}$, defined on different measure spaces $(S, \Sigma, \mu)$ and $\left(S^{\prime}, \Sigma^{\prime}, \mu^{\prime}\right)$, are (algebraically) conjugate when $\phi_{t}=\alpha \circ \phi_{t}^{\prime} \circ \alpha^{-1}$, where $\alpha$ is a measure-preserving transformation of $\Sigma$ onto $\Sigma^{\prime}$ with measure-preserving inverse $\alpha^{-1}$. This implies in particular that the measure-algebras $(S, \Sigma, \mu)$ and $\left(S^{\prime}, \Sigma^{\prime}, \mu^{\prime}\right)$ are isomorphic.

A $\sigma$-subalgebra $\Sigma^{\prime \prime}$ of $\Sigma$ is said to be invariant under a flow $\phi_{t}$ on $(S, \Sigma, \mu)$, whenever $\phi_{t}(E) \in \Sigma^{\prime \prime}$ for every $E \in \Sigma^{\prime \prime}$ and for every $t$. If $\Sigma^{\prime \prime}$ is an invariant $\sigma$-subalgebra of $\phi_{t}$, then clearly one obtains from $\phi_{t}$ a new flow $\phi_{t}^{\prime \prime}$ on the probability space $\left(S, \Sigma^{\prime \prime}, \mu\right)$ by restricting $\phi_{t}$ to the $\sigma$-subalgebra $\Sigma^{\prime \prime}$. The question stated at the end of $\$ 1$ can now be reformulated as follows: what other flows can one obtain (to within conjugacy) from a given flow $\phi_{t}$, by restricting $\phi_{t}$ to each one of its proper invariant $\sigma$-subalgebras? If the restriction $\phi_{t}^{\prime \prime}$ of a flow $\phi_{t}$ to some one of its invariant $\sigma$-subalgebras $\Sigma^{\prime \prime}$ is conjugate to another flow $\phi_{t}^{\prime}$, we shall say that $\phi_{t}$ is a model for $\phi_{t}^{\prime}$.

3. Our main result is then the following

TheOREM. Let $(S, \Sigma, \mu)$ be a separable nonatomic probability space. Then there exists a universal periodic flow $\Phi_{t}$ in $(S, \Sigma, \mu)$, namely, $a$ flow which is a model for every other periodic flow.

Proof. Recall the definition of a nonatomic separable measurealgebra, as given in Halmos [3, pp. 168-173]. From the discussion in Halmos, the following facts are easily derived: (a) the measure 
algebras of any two nonatomic separable probability spaces are isomorphic; (b) the product of two (and, in fact, of a countably infinite number of) nonatomic separable probability spaces is again a nonatomic separable probability space. (c) In particular, if $(S, \Sigma, \mu)$ is as in the statement of the Theorem, then the measure algebra of the "cylinder" space $\left(C, \Sigma_{1}, \nu\right)$ is isomorphic to that of $(S, \Sigma, \mu)$. We shall write a point of $C$ as the pair $(t, s)$, where $s \in S$ and $-\infty<t<\infty$, agreeing to identify two points whose $t$-coordinates differ by an integer. We now define $\Phi_{t}^{\prime}$ on $\left(C, \Sigma_{1}, \nu\right)$ as follows: set $\Phi_{t}^{\prime}\left(E_{1}\right)$ $=\left\{(u, s) \mid(u+t, s) \in E_{1}\right\}$. It is obvious that $\Phi_{t}^{\prime}$ is a flow. Let $\Phi_{t}$ be a flow conjugate to $\Phi_{t}^{\prime}$ and defined on $(S, \Sigma, \mu)$. Such a flow exists by (c). We claim that $\Phi_{t}$ is the desired universal flow.

To show this, it suffices to show that $\Phi_{t}^{\prime}$ is a universal flow, or, more simply, by (a), that $\Phi_{t}^{\prime}$ is a model for every flow $\phi_{t}$ on $(S, \Sigma, \mu)$.

Given $\phi_{t}$, consider the family $\Sigma^{\prime \prime}$ of sets $E^{\prime \prime}=\left\{(t, s) \mid s \in \phi_{t}(E), E \in \Sigma\right\}$. It is clear that $\Sigma^{\prime \prime}$ is a $\sigma$-subalgebra of $\Sigma_{1}$, and since $\Sigma$ is nonatomic, so is $\Sigma^{\prime \prime}$. Furthermore, $\nu\left(E^{\prime \prime}\right)=\mu(E)$. Indeed, $\nu\left(E^{\prime \prime}\right)$ can be calculated by Fubini's theorem, using the fact that the measure $\nu$ is the product of Lebesgue measure $d t$ with the measure $\mu$. This gives $\nu\left(E^{\prime \prime}\right)$ $=\int_{0}^{1} \int_{S} \phi_{t}(E) d \mu d t$. The inner integral gives $\mu\left(\phi_{t}(E)\right)=\mu(E)$, by the measure-preserving property of $\phi_{t}$. Thus the double integral simplifies to $\nu\left(E^{\prime \prime}\right)=\int_{0}^{1} \mu\left(\phi_{t}(E)\right) d t=\int_{0}^{1} \mu(E) d t=\mu(E)$.

Now define $\alpha: \Sigma \rightarrow \Sigma^{\prime \prime}$ as $\alpha(E)=E^{\prime \prime}$. Then $\alpha$ is an isomorphism of the measure-algebra of $(S, \Sigma, \mu)$ with that of $\left(C, \Sigma^{\prime \prime}, \nu\right)$. Let $\Phi_{i}^{\prime \prime}$ be the restriction of $\Phi_{t}^{\prime}$ to $\Sigma^{\prime \prime}$. To complete the proof of the theorem, we must establish that $\Sigma^{\prime \prime}$ is an invariant $\sigma$-subalgebra of $\Phi_{i}^{\prime}$ and that the diagram

$$
\begin{gathered}
\left(C, \Sigma^{\prime \prime}, \nu\right) \underset{\Phi_{t}^{\prime \prime}}{\longrightarrow}\left(C, \Sigma^{\prime \prime}, \nu\right) \\
\alpha \uparrow \alpha^{-1} \quad \alpha^{-1} \Downarrow \alpha \\
(S, \Sigma, \mu) \underset{\Phi_{t}}{\longrightarrow}(S, \Sigma, \mu)
\end{gathered}
$$

is commutative. But, for $E \in \Sigma$, we have by definition

$$
\begin{aligned}
\Phi_{i}^{\prime \prime}(\alpha(E)) & =\Phi_{t}^{\prime \prime}\left(E^{\prime \prime}\right)=\left\{(u, s) \mid(u+t, s) \in E^{\prime \prime}\right\} \\
& =\left\{(u, s) \mid s \in \phi_{u+t}(E)\right\}=\left\{(u, s) \mid s \in \phi_{u}\left(\phi_{t}(E)\right)\right\} .
\end{aligned}
$$

This shows that $\Phi_{t}^{\prime \prime}(\alpha(E))=\alpha\left(\phi_{t}(E)\right)$, or $\alpha^{-1} \circ \Phi_{t}^{\prime \prime} \circ \alpha=\phi_{t}$, and completes the proof.

4. Comments. The preceding result can be considered as a "strict sense" analog of a result obtained in the author's note [6]. It is 
clear that the result can be generalized to any nonseparable but homogeneous measure algebra. However, the statement is significant within the context of separable measure spaces, because it amounts to a restriction on the conjugacy classes of periodic flows.

We conclude with a number of questions suggested by the present situation, which would contribute to the solution of some current problems in ergodic theory.

(1) Suppose $\phi_{t}$ is a model for $\phi_{t}^{\prime}$ and $\phi_{t}^{\prime}$ is a model for $\phi_{t}$. What can be said about $\phi_{t}$ and $\phi_{t}^{\prime}$ ? Are they conjugate? If not, as is probable, then the equivalence relation thus obtained should be studied.

(2) What is the structure of the invariant $\sigma$-subalgebras of a measure-preserving transformation $\phi_{t}$ ?

(3) The usual "functorial" considerations suggest that there should be a dual result for a discrete group, that is, for a single measurepreserving transformation and its powers. In this direction, a method used by Oxtoby [5, p. 128], taking infinite direct products of probability spaces, is suggestive.

\section{BIBLIOGRAPHY}

1. Dunford-Schwartz, Linear operators, Vol. I, Interscience, New York, 1951.

2. Halmos, Lectures on ergodic theory, The Mathematical Society of Japan, Tokyo, 1956.

3. Halmos, Measure theory, Van Nostrand, New York, 1950.

4. Jacobs, Neuere Methoden und Ergebnisse der Ergodentheorie, Springer, Berlin, 1960.

5. Oxtoby, Ergodic sets, Bull. Amer. Math. Soc. 58 (1952), 116-136.

6. Rota, On models for linear operators, Comm. Pure Appl. Math. 13 (1960), 469-472.

Massachusetts Institute of Technology 